\title{
Commensurate Registry and Chemisorption at a Hetero-organic Interface
}

\author{
Benjamin Stadtmüller, ${ }^{1,2, *}$ Tomoki Sueyoshi, ${ }^{1,2}$ Georgy Kichin, ${ }^{1,2}$ Ingo Kröger, ${ }^{1,2, \dagger}$ Sergey Soubatch, ${ }^{1,2}$ \\ Ruslan Temirov, ${ }^{1,2}$ F. Stefan Tautz, ${ }^{1,2}$ and Christian Kumpf ${ }^{1,2}$ \\ ${ }^{1}$ Peter Grünberg Institut (PGI-3), Forschungszentrum Jülich, 52425 Jülich, Germany \\ ${ }^{2}$ Jülich Aachen Research Alliance (JARA)_Fundamentals of Future Information Technology, 52425 Jülich, Germany
} (Received 24 October 2011; published 6 March 2012)

We present evidence for a partly chemisorptive bonding between single monolayers of copper-IIphthalocyanine $(\mathrm{CuPc})$ and 3,4,9,10-perylene-tetracarboxylic-dianhydride (PTCDA) that are stacked on $\mathrm{Ag}(111)$. A commensurate registry between the two molecular layers and the substrate, i.e., a common crystallographic lattice for CuPc and PTCDA films as well as for the $\operatorname{Ag}(111)$ surface, indicates that the growth of the upper layer is dominated by the structure of the lower. Photoemission spectroscopy clearly reveals a gradual filling of the lowest unoccupied molecular orbital of PTCDA due to CuPc adsorption, which proves the chemisorptive character.

DOI: 10.1103/PhysRevLett.108.106103

The applicability of organic molecules as active materials in electronic devices such as organic light-emitting diodes, field-effect transistors, or solar cells depends not only on the properties of the bulk material itself, but also on the interfaces formed by the contact between different materials. In particular, electronic properties of interfaces show crucial impact on the overall performance of devices, which motivated intensive studies of organic-metal contacts in the last decades. A fundamental understanding of the key properties has been obtained from investigating model systems like 3,4,9,10-perylene-tetracarboxylicdianhydride (PTCDA) (see, e.g., [1-5]), metal phthalocyanines (MePc) ([6-9]), and other $\pi$-conjugated molecules adsorbing on single crystal surfaces [10-12]. However, regarding organic-organic heterojunctions, only a small number of examples can be found in the literature [13-18], even though such interfaces are at least of similar importance for organic electronic devices. It is also commonly believed that interfaces between different organic materials are generally dominated by very weak, van der Waals interaction, an assumption that will be proven to be incorrect.

We have investigated the geometric and electronic structure of the hetero-organic interface between the prototypical molecules copper-II-phthalocyanine $(\mathrm{CuPc})$ and PTCDA. These molecules were selected because their individual adsorption behaviors on $\operatorname{Ag}(111)$ are very different. While PTCDA tends to form islands due to attractive intermolecular forces [1-3], many phthalocyanines show repulsive interaction and hence form diluted, homogeneous phases in the submonolayer range $[8,9,19,20]$. The heterojunction is formed by adsorbing CuPc molecules on top of a closed layer of PTCDA on $\operatorname{Ag}(111)$. (See the Supplemental Material [21] for details on the sample preparation.) Figure 1 shows high-resolution low-energy electron-diffraction (LEED) images obtained with a spotprofile analysis (SPA) LEED instrument which give an
PACS numbers: 68.43.- h, 61.05.jh, 73.20.At, 79.60.Dp

overview of different phases that occur at $\mathrm{CuPc}$ coverages below one monolayer (ML) and at different temperatures. The well-known diffraction pattern of the PTCDA ML structure [dark spots in Fig. 1(a)] is equally visible in all images, indicating that the order in the PTCDA layer is not lifted by adsorption of CuPc.

With increasing $\mathrm{CuPc}$ coverage, new diffraction features arise at room temperature (RT): At first, between 0.3 and $0.5 \mathrm{ML}$, diffuse diffraction intensity is found around the (00) spot, indicating the lack of long-range order in the $\mathrm{CuPc}$ layer [Fig. 1(a)]. This diffuse intensity condenses

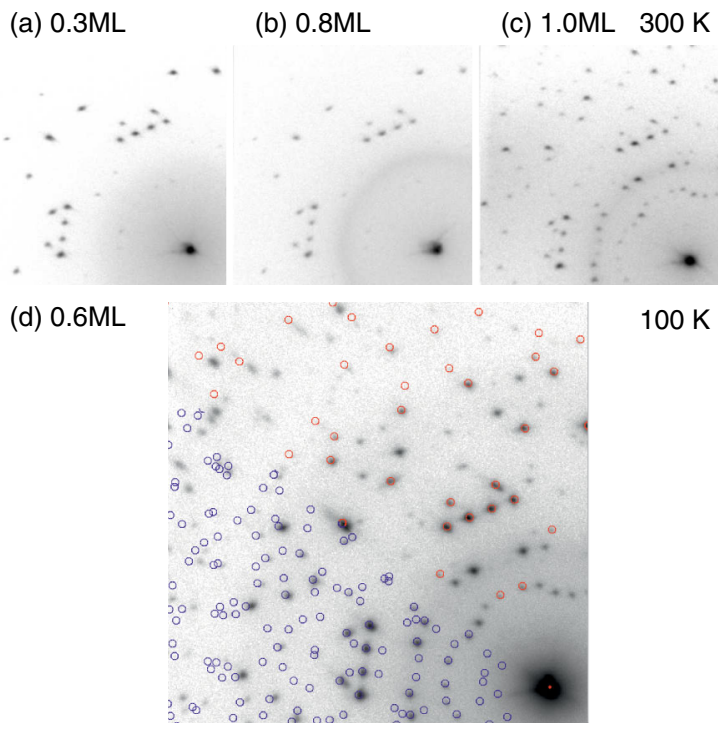

FIG. 1 (color online). High-resolution LEED images $\left(E_{\text {kin }}=\right.$ $27.2 \mathrm{eV}$ ) for different $\mathrm{CuPc}$ coverages adsorbed on a closed layer PTCDA/Ag(111). Images in panels (a), (b), and (c) were recorded at RT; the image in panel (d) was recorded at $100 \mathrm{~K}$. Red and blue circles mark calculated positions of diffraction spots from the PTCDA-monolayer structure and the $\mathrm{CuPc} /$ PTCDA stacked-bilayer (SB) structure, respectively. 
into a homogenous ring which is visible between 0.5 and 1.0 ML [Fig. 1(b)]. The radius of the ring, which corresponds to the average distance between the molecules, increases continuously with increasing coverage and indicates a more and more densely packed $\mathrm{CuPc}$ layer and a correspondingly shrinking intermolecular distance $[9,20]$. When the first CuPc layer is (almost) closed, the LEED pattern changes. The homogeneous ring vanishes and sharp diffraction spots appear in addition to the spots from the PTCDA structure [Fig. 1(c)]. They are visible up to rather high orders of diffraction and indicate the condensation of the $\mathrm{CuPc}$ molecules into a well-ordered structure, which we call stacked-bilayer (SB) structure in the following.

The same long-range ordered structure is observed in a wide range of $\mathrm{CuPc}$ coverages $\theta$ upon cooling the sample [0.4 ML $<\theta<1.0 \mathrm{ML}, T<160 \mathrm{~K}$; see Fig. 1(d)]. This observation indicates that the SB structure corresponds to the global minimum of the total interface potential. Its formation is induced either by cooling (i.e., by reducing thermal motion of the molecules) or by increasing the coverage (i.e., by sterically hindering diffusion of the molecules). Hence, the behavior of CuPc on PTCDA/ $\operatorname{Ag}(111)$ is rather similar to its adsorption on the clean $\mathrm{Ag}(111)$ [9] or the $\mathrm{Au}(111)$ surface [20]. In particular, at $\mathrm{RT}$, no island formation is observed, which indicates a very weak or even repulsive interaction between the CuPc molecules. On the other hand, condensation of the CuPc molecules to well-ordered islands at low temperature indicates some interaction with the underlying PTCDA layer or even with the Ag surface, as we will discuss later.

The LEED pattern of the SB phase can be explained unambiguously by one single structural model. In the upper right half of Fig. 1(d), the PTCDA diffraction spots are marked by red circles, while in the lower left, the spots stemming from the $\mathrm{CuPc}$ cell are indicated in blue. Deviations between measured and calculated spot positions at high $k$ values are due to rather extreme trajectories of the diffracted electron beams passing through the inhomogeneous electric field close to the octupole electrodes of the SPA-LEED instrument, and hence they represent an instrumental artefact.

Note that all PTCDA spots can also be indexed as CuPc spots. This immediately shows that the structure of the $\mathrm{CuPc}$ overlayer is commensurate with the PTCDAmonolayer structure and explains the rather high intensity of the high-order diffraction spots in the LEED image. Consequently, the superstructure matrices describing the unit cell of the $\mathrm{CuPc}$ and PTCDA stacked-bilayer structure have integer numbers only [22]:

$$
\left(\begin{array}{l}
\vec{A} \\
\vec{B}
\end{array}\right)_{\mathrm{SB}}=\left(\begin{array}{cc}
3 & 1 \\
-2 & 1
\end{array}\right)\left(\begin{array}{l}
\vec{A} \\
\vec{B}
\end{array}\right)_{\mathrm{PTCDA}}=\left(\begin{array}{cc}
3 & 16 \\
-12 & -9
\end{array}\right)\left(\begin{array}{l}
\vec{A} \\
\vec{B}
\end{array}\right)_{\mathrm{Ag}} .
$$

To our knowledge, a commensurate registry between two different organic monolayer structures has not been reported before. It of course also means that the second organic layer $(\mathrm{CuPc})$ in this system is still commensurate with the $\mathrm{Ag}(111)$ substrate. The appearance of commensurate structures is usually an indication of a relatively strong interaction of organic molecules with the underlying substrate. Commensurability across an organic-organic heterojunction is therefore very unexpected and stands in contrast to the structure formation usually reported for such interfaces [15-18,23]. Even the same molecules on a different substrate (CuPc and PTCDA on HOPG) behave differently: Periodic displacement lines occur in the CuPc layer, caused by a lattice mismatch between the $\mathrm{CuPc}$ and the PTCDA layer [16]. However, in our case, commensurability is a very characteristic feature with important consequences for the formation of the CuPc film: In contrast to the almost square unit cells generally found for $\mathrm{CuPc}$, and rectangular cells for PTCDA (both reflecting the fourfold or twofold symmetry of the molecule), the SB structure has an oblique unit cell with the dimensions $\left|\vec{A}_{\mathrm{SB}}\right|=42.6 \AA$, $\left|\vec{B}_{\mathrm{SB}}\right|=31.3 \AA$, and $\gamma=116.26^{\circ}$. This indicates that commensurability is not just the result of an arbitrary coincidence of similar lattices of the isolated PTCDA and $\mathrm{CuPc}$ layers. There must be a significant interaction across the heterojunction that dominates the structure formation and forces the CuPc layer into an unusual structure that otherwise would not be formed.

We used low-temperature scanning tunneling microscopy (STM) to investigate this system further and determine positions and orientations of the molecules in the unit cell. Figure 2 presents raw-data constant-current images recorded with a Createc STM at $10 \mathrm{~K}$. Figure 2(a) shows the edge of a CuPc island on the PTCDA monolayer with submolecular resolution in both regions: the PTCDA monolayer (lower part) and the CuPc island on top (upper part). It can be seen that $\mathrm{CuPc}$ homogeneously overgrows the PTCDA monolayer. The unit-cell dimensions obtained from SPA-LEED are confirmed by the STM images. Translating the well-known PTCDA superlattice [indicated by the red grid in the lower part of Fig. 2(a)] onto the CuPc island in the upper part and comparing it with the unit cell of that region (indicated by the blue grid) illustrates matching of the periodicities of the SB and PTCDA-monolayer phases.

Figure 2(b) shows a close-up of the CuPc molecules. Ball-and-stick models indicate six molecules within one SB unit cell. They all show the typical crosslike STM contrast, even though their wings differ in apparent height. In Fig. 2(c), the positions of the underlying PTCDA molecules are marked as dotted ellipses. This plot reveals that the $\mathrm{CuPc}$ wings that appear bright are located directly above the PTCDA molecules, while dark wings are bridging the PTCDA molecules; i.e., they lie above the "gaps" in the lower layer. This finding indicates that the different contrast of the molecular wings is caused by an electronic coupling between the first and second molecular layers. It appears as if the underlying PTCDA molecule facilitates 
electronic transport through that part of the $\mathrm{CuPc}$ molecule that lies exactly above the central part of the PTCDA molecule.

A closer look at the structural details shows that-apart from the different brightness of the wings- the six CuPc molecules are equivalent: They are equally oriented with an angle of $45^{\circ} \pm 2^{\circ}$ relative to the [110] direction of the silver substrate and form a square lattice with a spacing of $\approx 14 \AA$, which also equals the intermolecular distance. This reflects the size as well as the fourfold symmetry of the molecule and matches the structural parameters that are typically found for a densely packed layer of CuPc molecules $[9,20]$. We observed three different adsorption sites for CuPc on the PTCDA layer, each occupied twice per unit cell. Figure 2(d) shows a model of the PTCDA layer, and red circles mark the adsorption sites. The size of the circles corresponds to the statistical error. The center of the $\mathrm{CuPc}$ molecule is either located on top of the PTCDA backbone [PTCDA top site, labeled " $T$ " in Fig. 2(d)] or above the ditch between either two (bridge site, " $B$ ") or three PTCDA molecules (hollow site, " $H$ "). The mismatch of the rectangular structure of PTCDA/Ag(111) and the fourfold symmetry of the CuPc molecule (which suggests a squared structure) does not allow that all molecules adsorb

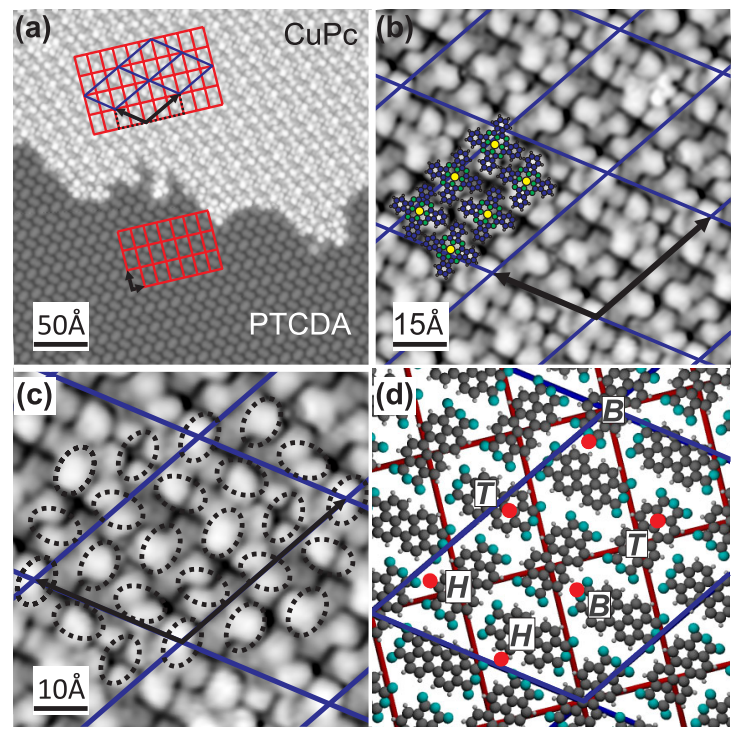

FIG. 2 (color online). Panels (a), (b), and (c): STM images of the $\mathrm{SB}$ phase at $T=10 \mathrm{~K}(0.75 \mathrm{~V}, 18 \mathrm{pA})$. All images were corrected for lateral distortions using the well-known PTCDA-on- $\mathrm{Ag}(111)$ unit cell. (a) A CuPc island (bright contrast) has condensed on a closed PTCDA monolayer on $\operatorname{Ag}(111)$. PTCDA and CuPc lattices are indicated by red and blue lines, respectively. (b) Close-up of the $\mathrm{CuPc}$ island. Six CuPc molecules (one unit cell) are shown as ball-and-stick models. (c) Dotted ellipses indicate the positions of PTCDA molecules under the $\mathrm{CuPc}$ layer. Bright STM contrast is found where $\mathrm{CuPc}$ wings lie above the PTCDA core. Panel (d) Model of the PTCDA ML-structure. Adsorption sites of CuPc molecules are marked by red circles, the size of which indicate statistical errors. on identical sites and hence inhibits the densest possible packing of the $\mathrm{CuPc}$ molecules. Obviously, the observed arrangement represents the best compromise between adsorbing as many $\mathrm{CuPc}$ molecules as possible (forming the densest packing possible) and obtaining the energetically best adsorption sites. This indicates that the modulations of the interface potential must be rather large; otherwise, no commensurate configuration but rather a (more densely packed) point-on-line or incommensurate structure would be formed. The reason for this unexpected finding, which is at variance with many others reported before [15-18], will now be discussed on the basis of $\mathrm{x}$-ray standing waves and ultraviolet photoelectron spectroscopy (UPS) results.

With x-ray standing waves, the vertical structure, i.e., the adsorption heights of individual atomic species, can be measured with very high precision. We found that the wellknown adsorption height of PTCDA/Ag(111) (2.86 § [4]) is only weakly affected by the additional adsorption of $\mathrm{CuPc}$; it changes to $2.81 \AA$. The $\mathrm{CuPc}$ layer we find at a height of $6.03 \AA$, which is only $3.22 \AA$ above the PTCDA layer. These numbers indicate that the adsorption height of $\mathrm{CuPc}$ on PTCDA/Ag(111) lies between the values for $\mathrm{CuPc} / \mathrm{Ag}(111)(3.09 \AA$ [9]) and $\mathrm{CuPc} / \mathrm{Au}(111)(3.31 \AA$ [24]). These two systems represent typical examples for chemisorption and physisorption, respectively. Hence, we can conclude that $\mathrm{CuPc}$ is not purely physisorbed on PTCDA/Ag(111). It should further be mentioned that the atomic species of the $\mathrm{CuPc}$ molecule are all found within less than $0.1 \AA$ in height; i.e., the CuPc molecules are not significantly bent or distorted. Hence, the $\mathrm{Cu}$ metal atom obviously does not play a prominent role in the heteroepitaxial interaction. This conclusion is also supported by unremarkable $\mathrm{Cu}$ x-ray photoemission spectra in which no core-level shifts could be detected. Further details will be published in a subsequent paper.

We also used UPS to gain more insight into the electronic interaction between the substrate and the molecular layers. Figure 3 shows He I $\alpha$ UPS data recorded for different submonolayer $\mathrm{CuPc}$ coverages between 0 and $0.9 \mathrm{ML}$ at RT using a Scienta R4000 electron analyzer. In the spectra of the bare PTCDA monolayer (lowest curves, labeled "0.0 ML"), the highest occupied molecular orbital (HOMO) and a second state close to the Fermi level, the former lowest unoccupied molecular orbital (F-LUMO), can be seen. The F-LUMO is partially filled due to charge transfer from the substrate into the molecule and directly indicates a chemisorptive interaction between the $\operatorname{Ag}(111)$ substrate and the PTCDA molecule [2,25,26]. This wellknown effect occurs upon chemisorption of many organic molecules on noble metal surfaces $[1,2,7,10,20]$. Note that different intensities of the HOMO peak for both emission angles are caused by the characteristic photoemission angular dependence of the PTCDA HOMO [27,28].

Upon adsorption of $\mathrm{CuPc}$ on top of the PTCDA layer, two new peaks appear in the spectra, the HOMO and 


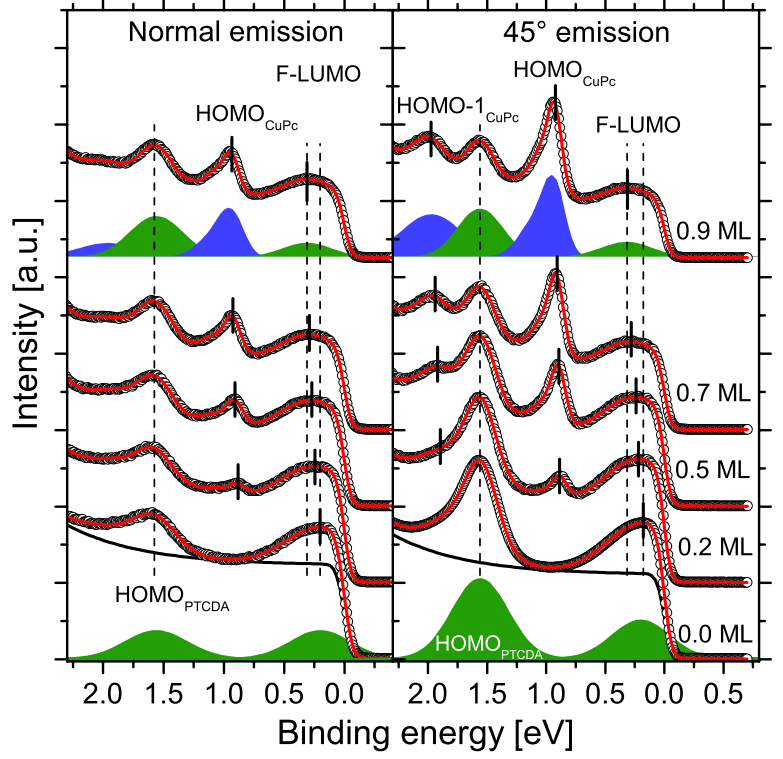

FIG. 3 (color online). UPS data of CuPc and PTCDA on $\mathrm{Ag}$ (111) for different $\mathrm{CuPc}$ coverages at RT (overlapping black circles along the curves, $\hbar \omega=21.218 \mathrm{eV}$, photoelectron acceptance angle $30^{\circ}$ ). The red curves represent least-square fits to the data. For the lowermost and uppermost curves, the fitting model consisting of up to four independent peaks is shown; PTCDA and $\mathrm{CuPc}$ states are displayed in green and blue, respectively. For fitting the background, we used a Fermi function which was broadened in order to account for the instrumental resolution, plus an exponential component. It is shown as a black solid line with the lowermost curve.

HOMO-1 states of CuPc. All features in the spectra are fitted with single Gaussian peaks, except the CuPc HOMO peak, which is known to have an asymmetric shape and hence requires a progression of two Gaussians. At a coverage of $0.2 \mathrm{ML}$, the $\mathrm{CuPc} \mathrm{HOMO}$ is found at a binding energy of $E_{\mathrm{B}}=0.88 \mathrm{eV}$, but shifts significantly (by $40 \mathrm{meV}$ ) to higher binding energies at higher coverage (0.9 ML). It is identified by its asymmetric shape [29] and is easily seen for both emission angles. The HOMO- 1 state can be seen clearly only under off-normal emission. It appears as a shoulder of the PTCDA HOMO-peak at $E_{\mathrm{B}}=$ $1.90 \mathrm{eV}$, but becomes easier to see at higher coverage due to a shift of $+80 \mathrm{meV}$.

However, the more important change in the spectra caused by the adsorption of $\mathrm{CuPc}$ is a shift of the F-LUMO state of PTCDA. As illustrated by the vertical dashed lines in Fig. 3, this state shifts continuously from $E_{\mathrm{B}}=0.20 \mathrm{eV}$ to $E_{\mathrm{B}}=0.32 \mathrm{eV}$, i.e., by as much as $120 \mathrm{meV}$. This shift is significant with respect to both instrumental resolution $(30 \mathrm{meV})$ and width of the peak (between 0.45 and $0.55 \mathrm{eV}$, depending on coverage). It indicates an additional filling of the PTCDA F-LUMO induced by the adsorption of CuPc molecules, i.e., an increase of the charge transfer between substrate and adsorbate (and/or between both organic layers). This increase implies an enhancement of the chemisorptive interaction between the CuPc and PTCDA adsorbate layers and the substrate, and is in strong contrast to previous studies which reported only very weak interactions across organicorganic interfaces $[13,15,17]$. It is remarkable that the PTCDA HOMO state does not shift significantly. This finding might be an indication that the CuPc LUMO may be more strongly involved in the additional charge transfer than the PTCDA F-LUMO. In such case, the F-LUMO peak in the spectra should have two components, although they cannot clearly be verified in the data. Thus, the nonshifting HOMO might also be accidental, since frontier orbitals are often affected very differently by electronic-charge transfer.

In conclusion, we have presented evidence for an unexpectedly strong interaction (namely, a weak chemisorption) at the organic heterojunction between $\mathrm{CuPc}$ and PTCDA on $\operatorname{Ag}(111)$. The chemisorptive character manifests itself in several direct and indirect findings. (1) The structure formation of the CuPc layer is clearly dominated by the structure of the underlying PTCDA layer. This formation is indicated by an unusual unit-cell shape that does not reflect the symmetry or size of the CuPc molecules and-more impressively-by the formation of a commensurate registry between the $\mathrm{CuPc}$ and PTCDA superstructures [and also with the $\operatorname{Ag}(111)$ surface]. The commensurate organic-organic heteroepitaxial interface was consistently found by SPA-LEED and STM. (2) STM images reveal that the contrast of the benzene wings of the $\mathrm{CuPc}$ molecules depends strongly on their position relative to the underlying PTCDA molecule, which is clearly not a geometric but an electronic effect stemming from the interaction between the two types of molecules. This is also indicated by XSW results, which reveal flat-lying $\mathrm{CuPc}$ molecules at an interlayer distance corresponding to a weak chemisorptive interaction between the organic layers. (3) The interaction unmasks itself as charge-transfer effect in UPS. The F-LUMO state of PTCDA, which is already partly filled due to the adsorption on the $\operatorname{Ag}(111)$ surface, is gradually filled up further upon adsorption of $\mathrm{CuPc}$. This is evidenced by a large shift $(120 \mathrm{meV})$ of the F-LUMO toward the high binding-energy side. Possibly, the LUMO of the CuPc molecules is also involved in the charge transfer.

Against common belief, our results prove that rather strong, chemisorptive interaction can occur also at hetero-organic interfaces. This aspect will have significant impact on the understanding and designing of molecular electronic devices.

*b.stadtmueller@fz-juelich.de

${ }^{\dagger}$ Present address: Physikalisch-Technische Bundesanstalt, Bundesallee 100, 38116 Braunschweig, Germany.

[1] S. Duhm, A. Gerlach, I. Salzmann, B. Bröker, R. Johnson, F. Schreiber, and N. Koch, Org. Electron. 9, 111 (2008). 
[2] Y. Zou, L. Kilian, A. Schöll, T. Schmidt, R. Fink, and E. Umbach, Surf. Sci. 600, 1240 (2006).

[3] F. S. Tautz, Prog. Surf. Sci. 82, 479 (2007).

[4] A. Hauschild, R. Temirov, S. Soubatch, O. Bauer, A. Schöll, B. C. C. Cowie, T.-L. Lee, F. S. Tautz, and M. Sokolowski, Phys. Rev. B 81, 125432 (2010).

[5] I. Kröger, B. Stadtmüller, C. Wagner, C. Weiss, R. Temirov, F. S. Tautz, and C. Kumpf, J. Chem. Phys. 135, 234703 (2011).

[6] T. Kataoka, H. Fukagawa, S. Hosoumi, K. Nebashi, K. Sakamoto, and N. Ueno, Chem. Phys. Lett. 451, 43 (2008).

[7] M. Häming, C. Scheuermann, A. Schöll, F. Reinert, and E. Umbach, J. Electron Spectrosc. Relat. Phenom. 174, 59 (2009).

[8] C. Stadler, S. Hansen, I. Kröger, C. Kumpf, and E. Umbach, Nature Phys. 5, 153 (2009).

[9] I. Kröger, B. Stadtmüller, C. Stadler, J. Ziroff, M. Kochler, A. Stahl, F. Pollinger, T.-L. Lee, J. Zegenhagen, F. Reinert, and C. Kumpf et al., New J. Phys. 12, 083038 (2010).

[10] F. Schreiber, Prog. Surf. Sci. 65, 151 (2000).

[11] C. Stadler, S. Hansen, A. Schöll, T.-L. Lee, J. Zegenhagen, C. Kumpf, and E. Umbach, New J. Phys. 9, 50 (2007).

[12] J. Stanzel, W. Weigand, L. Kilian, H. Meyerheim, C. Kumpf, and E. Umbach, Surf. Sci. 571, L311 (2004).

[13] M. Häming, M. Greif, C. Sauer, A. Schöll, and F. Reinert, Phys. Rev. B 82, 235432 (2010).

[14] I. G. Hill, D. Milliron, J. Schwartz, and A. Kahn, Appl. Surf. Sci. 166, 354 (2000).

[15] S. C. Mannsfeld, K. Leo, and T. Fritz, Phys. Rev. Lett. 94, 056104 (2005).

[16] W. Chen, H. Huang, S. Chen, X. Y. Gao, and A. T. S. Wee, J. Phys. Chem. C 112, 5036 (2008).
[17] F. Sellam, T. Schmitz-Hübsch, M. Toerker, S. Mannsfeld, H. Proehl, T. Fritz, K. Leo, C. Simpson, and K. Müllen, Surf. Sci. 478, 113 (2001).

[18] H. Huang, W. Chen, S. Chen, D. C. Qi, X. Y. Gao, and A. T. S. Wee, Appl. Phys. Lett. 94, 163304 (2009).

[19] C. Stadler, S. Hansen, F. Pollinger, C. Kumpf, E. Umbach, T.-L. Lee, and J. Zegenhagen, Phys. Rev. B 74, 035404 (2006).

[20] B. Stadtmüller, I. Kröger, F. Reinert, and C. Kumpf, Phys. Rev. B 83, 085416 (2011).

[21] See the Supplemental Material at http://link.aps.org/ supplemental/10.1103/PhysRevLett.108.106103 for details on the sample preparation.

[22] The unit-cell vectors of the PTCDA cell $(\vec{A}, \vec{B})_{\mathrm{PTCDA}}$ correspond to the PTCDA supercell $(35 \mid-61)$, which describes one of the mirror domains of the more commonly used matrix ( $71 \mid 25)$.

[23] C. Wagner, R. Forker, and T. Fritz, J. Phys. Chem. Lett. 3, 419 (2012).

[24] I. Kröger, B. Stadtmüller, C. Kleimann, P. Rajput, and C. Kumpf, Phys. Rev. B 83, 195414 (2011).

[25] R. Temirov, S. Soubatch, A. Lucian, and F. S. Tautz, Nature (London) 444, 350 (2006).

[26] M. Rohlfing, R. Temirov, and F. S. Tautz, Phys. Rev. B 76, 115421 (2007).

[27] J. Ziroff, F. Forster, A. Schöll, P. Puschnig, and F. Reinert, Phys. Rev. Lett. 104, 233004 (2010).

[28] P. Puschnig, E.-M. Reinisch, T. Ules, G. Koller, S. Soubatch, M. Ostler, L. Romaner, F.S. Tautz, C. Ambrosch-Draxl, and M. G. Ramsey, Phys. Rev. B 84, 235427 (2011).

[29] F. Evangelista, V. Carravetta, G. Stefani, B. Jansik, M. Alagia, S. Stranges, and A. Ruocco, J. Chem. Phys. 126, 124709 (2007). 